\title{
Grey Correlation Coefficient Analysis and Estimation of Urban Living Garbage in Harbin
}

\author{
Lihong Sun ${ }^{1, a^{*}}$, Yu Zhang ${ }^{1}$ \\ ${ }^{1}$ The basic science college of Harbin university of commerce, Harbin 150028 Heilongjiang China \\ ahonghong03050305@163.com
}

Keywords: municipal solid waste, collected amount, correlation analysis, Grey Model.

Abstract. The amount of garbage conforms to the gray data characteristics because of the uncertainty and dispersion of urban living garbage produced, the corresponding dynamic prediction model based on Grey model can be established due to the uncertainty and dispersion of the collected amount in line with Grey feature. First the method of Grey correlation coefficient analysis was used to quantitatively analyze the impacts of waste generation factors, the result shows that the first order of the precision is per capita consumption expenditure; Second the gray GM(1.1) prediction model was established to predict the amount of garbage produced in Harbin for the next few years. The result is helpful for the management and the planning of Harbin.

\section{Introduction}

In our country, with the rapid development of urban economy, the social problems and the environment problem caused by urban living garbage are increasingly highlighted, discharge of municipal solid waste is the important basis of planning and construction of urban environment health, the prediction of Municipal solid waste output is one of the important reference index accepted as city life garbage disposal and transportation development planning and urban development and planning, it's an important fundamental research work. The uncertainty and dispersion by urban living garbage producing makes its QingYunLiang to conform to the characteristics of grey data ${ }^{[1]}$, the Urban living garbage output can use gray theory to analyze and set up a corresponding grey model to forecast.

\section{Grey Relational Analysis}

Grey relational coefficient and correlation degree. Correlation degree was used to describe degree of correlation of two systems in the process of development and changes. Using grey correlation analysis method can analyze more accurately the correlation between the two systems through a small amount of data. T correlation coefficient was used to Compare the close degree of two sequences $^{[2]}$. let $Y_{0}(t), t=1,2, \cdots n$ to be the mother series for Garbage output, $Y_{i}(t)(i=1,2, \cdots m)$ is the subsequence, the correlation coefficient of the subsequence comparing $Y_{i}(t)$ to the mother series $Y_{0}(t)$ at $t$ time is:

$$
\zeta_{i}(t)=\frac{\min \min \left|y_{0}(t)-y_{i}(t)\right|+\rho \max \max \left|y_{0}(t)-y_{i}(t)\right|}{\left|y_{0}(t)-y_{i}(t)\right|+\rho \max \max \left|y_{0}(t)-y_{i}(t)\right|}
$$

Among $\min \min \left|y_{0}(t)-y_{i}(t)\right| \mathrm{t} \max \max \left|y_{0}(t)-y_{i}(t)\right|$ he minimum differential and the biggest difference between two levels, $\rho$ is resolution, $0<\rho<1$ and usually take $\rho=0.5$. According to the correlation coefficient formula of the above equation (1), the correlation degree of two sequences is

$$
R_{i}=\frac{1}{n} \sum_{t=1}^{n} \zeta_{i}(t)
$$

Correlation degree analysis about Harbin city life garbage output. Using correlation analysis method to analyze urban living garbage, several indicators ${ }^{[3]}$ were selected generally from the 
population, urban construction and economic factors, then collecting the relevant historical data in Harbin, specific data was listed in table 1: According to equation (1) and (2) grey correlation degree was calculated, the results were shown in table 2:

Table 1 Harbin city life garbage output and each influence factor from 2004 to 2013

\begin{tabular}{lclcccc}
\hline year & $\begin{array}{c}\text { garbage } \\
\text { output }\end{array}$ & GDP & population & City gas rate & $\begin{array}{l}\text { Total retail sales of } \\
\text { consumer goods }\end{array}$ & $\begin{array}{l}\text { Annual per capita } \\
\text { consumption expenditure }\end{array}$ \\
\hline 2004 & 98 & 646.13 & 688.01 & 81.51 & 360.42 & 5445.7 \\
2005 & 131.6 & 733.85 & 694.84 & 85.43 & 365.93 & 5815.66 \\
2006 & 126 & 823.5 & 702.59 & 87.52 & 409.39 & 6419.21 \\
2007 & 147 & 941.6 & 716.58 & 91.23 & 440.05 & 6805.3 \\
2008 & 133 & 1102.39 & 725.01 & 91.2 & 578.6 & 7427.82 \\
2009 & 145 & 1313.93 & 741.73 & 91.3 & 670.56 & 7899.81 \\
2010 & 119 & 1538.94 & 753.11 & 92.62 & 784.95 & 8986.87 \\
2011 & 147 & 1856.63 & 764.25 & 98.6 & 936.21 & 10097.95 \\
2012 & 152 & 2318.14 & 772.3 & 97.66 & 1176.58 & 12015.81 \\
2013 & 179 & 2724.08 & 781.67 & 98.15 & 1381.12 & 14250.78 \\
\hline
\end{tabular}

Table 2 correlation degree and rank of Five indicators and garbage output

\begin{tabular}{ccc}
\hline indicator & correlation degree & rank \\
\hline Annual per capita consumption expenditure of urban households & 0.697031 & 1 \\
Total retail sales of consumer goods & 0.632324 & 2 \\
GDP & 0.629015 & 3 \\
City gas rate & 0.627936 & 4 \\
population & 0.607275 & 5 \\
\hline
\end{tabular}

By the above result, the biggest influence on garbage output factor is the per capita consumption expenditure, followed by total retail sales of social consumer goods, GDP and so on, Thus it can be seen economic factors on behalf of the residents living standard are the main factors influencing the life garbage output.

GM $(1,1)$ Model $^{[3]}$ about Garbage Output. Let waste output before $k$ years to be the original series $x^{(0)}=\left(x^{(0)}(1), x^{(0)}(2), \cdots x^{(0)}(k)\right)$, grey model are defined as following differential equation:

$$
\frac{d x^{(1)}}{d t}+a x^{(1)}=u
$$

Among $x^{(1)}(k)$ is the accumulation generation sequence of $x^{(0)}(k), x^{(1)}(k)=\sum_{i=1}^{k} x^{(0)}(i), a$ is called the development coefficient, $u$ is called grey action quantity. calculation of the parameters, $a, u$ of the model was used the least square method, the above model by discretization was expressed by matrix for:

$$
y_{N}=B P \text {. }
$$

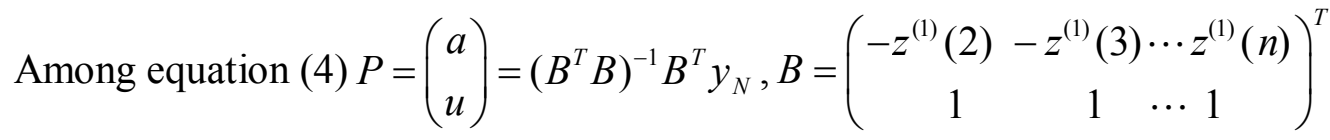

$$
y_{N}=\left(x^{(0)}(2), x^{(0)}(3) \cdots x^{(0)}(n)\right)^{T}, z^{(1)}(k)=0.5 x^{(1)}(k)+0.5 x^{(1)}(k-1), k=2,3, \cdots n
$$

The model of the continuous variable is

$$
x^{(1)}=\left(x^{(0)}(1)-\frac{u}{a}\right) e^{-a t}+\frac{u}{a}
$$


model by Discretization is

$$
x^{(1)}(k+1)=\left(x^{(0)}(1)-\frac{u}{a}\right) e^{-a k}+\frac{u}{a}, k=0,1,2, \cdots, n,
$$

After reduction the model is

$$
x^{(0)}(k+1)=e^{-a(k-1)}\left(x^{(0)}(1)-\frac{u}{a}\right)\left(e^{a}-1\right) .
$$

GM (1, 1) Model Prediction of Garbage Output on Harbin . Let garbage output data In 1997-2013 in Harbin to be the original sequence, as shown in table 1.Using the equation (4)-(7) to forecast Harbin city life garbage output ${ }^{[4]}$ and obtain the following models:

$$
x^{(1)}(k+1)=461.2346 e^{0.0187 k}-452.9946, \quad k=0,1,2, \cdots, n
$$

The results was shown in table 3 .

table 3 the actual and estimated values and the relative error of garbage output from 1997 to 2013 in Harbin

\begin{tabular}{llllllllll}
\hline year & 1997 & 1998 & 1999 & 2000 & 2001 & 2002 & 2003 & 2004 & 2005 \\
\hline estimated value $/ 10^{4} t$ & 8.24 & 8.71 & 8.87 & 9.03 & 9.21 & 9.39 & 9.56 & 9.74 & 9.92 \\
Actual value/ $10^{4} t$ & 8.24 & 8.55 & 8.95 & 9.16 & 9.27 & 9.39 & 9.49 & 9.68 & 9.87 \\
relative error $/ \%$ & 0.00 & 1.87 & -0.89 & -1.41 & -0.65 & 0.00 & 0.74 & 0.62 & 0.51 \\
\hline year & 2006 & 2007 & 2008 & 2009 & 2010 & 2011 & 2012 & 2013 & \\
\hline estimated value $/ 10^{4} t$ & 10.11 & 10.3 & 10.49 & 10.7 & 10.9 & 11.1 & 11.31 & 11.53 & \\
Actual value $/ 10^{4} t$ & 10.07 & 10.29 & 10.57 & 10.86 & 10.97 & 11.01 & 11.21 & 11.41 & \\
relative error $/ \%$ & 0.4 & 0.097 & -0.76 & -1.47 & -0.64 & 0.81 & 0.89 & 1.05 & \\
\hline
\end{tabular}

Precision Test . Grey model inspection methods commonly used posterior precision posterior difference test (the variance ratio). Namely, respectively, the variance of the original data sequence and residual error sequence $S_{1}, S_{2}$, let $C=\frac{S_{2}}{S_{1}}$ to be a posteriori difference test data. according to the formula $p=\left\{|e(k)-\bar{e}|<0.6745 S_{1}\right\}$ to calculate the Small error probability. the precision of the model was usually depicted by $\mathrm{c}$ and $\mathrm{p}$, and according to its value to classify the accuracy .The model precision grade standard was shown in table 4.

table 4 The model precision grade judging table

\begin{tabular}{ccc}
\hline model precision grade & $P$ & $C$ \\
\hline 1 & $p \geq 0.95$ & $C \leq 0.35$ \\
2 & $0.80 \leq p<0.95$ & $0.35<C \leq 0.50$ \\
3 & $0.70 \leq p<0.80$ & $0.50<C \leq 0.65$ \\
unqualified & $p<0.70$ & $C>0.65$ \\
\hline
\end{tabular}

Using residual analysis and a posteriori difference test the accuracy of the model, let the original data to be $x^{(0)}=\left(x_{1}^{(0)}, x_{2}^{(0)}, \cdots x_{n}^{(0)}\right)$, then the residual error is $e(k)=x^{(0)}(k)-\hat{x}^{(0)}(k)$, the mean of The original sequence is $\bar{x}^{(0)}=\frac{1}{17} \sum_{k=1}^{17} x_{k}^{(0)}=9.9406$, the average error is $\bar{e}=\frac{1}{17} \sum_{k=1}^{17} e(k)=-0.00706$, he variance of the original data sequence is $S_{1}^{2}=\frac{1}{17} \sum_{k=1}^{17}\left(x_{k}^{(0)}-\bar{x}^{(0)}\right)^{2}=0.8705$, variance of residual error sequence is 
$S_{2}^{2}=\frac{1}{17} \sum_{k=1}^{17}(e(k)-\bar{e})^{2}=0.0081$, so $C=\frac{S_{2}}{S_{1}}=0.09626, p=\left\{|e(k)-\bar{e}|<0.6745 S_{1}\right\}$

According to table 4 the model precision ${ }^{[5]}$ is grade 1 .According to this model to predict the waste output with 2015-2020, the results were shown in table 5, in the next few years the output will be a trend of increasing year by year.

table 5 Harbin garbage output prediction value In 2015-2020

\begin{tabular}{lllllll}
\hline year & 2015 & 2016 & 2017 & 2018 & 2019 & 2020 \\
\hline Prediction value/ $/ 0^{4} t$ & 11.96 & 12.19 & 12.42 & 12.65 & 12.89 & 13.13 \\
\hline
\end{tabular}

\section{Conclusion.}

Per capita consumption expenditure is the greatest impact on Harbin city life rubbish emission through the calculation of grey correlation degree, followed by total retail sales of social consumer goods, so the economic factor on behalf of the residents living standard is the main factor that influence the production of household waste output. using GM $(1,1)$ model to forecast the Harbin city garbage output, model prediction precision and prediction accuracy is higher, the Harbin city life garbage output in the coming years will be a trend of increasing year by year.

\section{Acknowledgement}

Fund project: heilongjiang province education department project (12531170).

\section{References}

[1] Sifeng Liu, Naiming Xie and so on. The grey system theory and applications (the seventh edition) [M]. Beijing: science press,2014,28-35..

[2] Youqing Yang, Yongquan Yuan, Jie Sun.Factor Analysis and Grey Model Predication of Domestic Refuse Yields in Qingdao City[J]Journal of South-Central University for Nationalities(Nat Sci Edition), 2005,24(3):5-8.

[3] Tao Yu, Tao Huang and so on. Multi-variate statistical analysis of influential factors of the delivering quantity of municipal solid waste in Lanzhou city[J]. Journal of Guangxi University:Nat Sci Ed, 2013,38(2):500-506.

[4] Jie Kang, Bei Guo. Analysis on Influencing Factors and Grey Model of Municipal Domestic Waste in Xi'an City[J].ENVIRONMENTAL SCIENCE AND MANAGEMENT,2011,36(12):51-54.

[5] Wei Chu, Shiqun Sun and so on. Study on Grey Correlation Analysis and Prediction of Municipal Solid Waste in Hef ei City[J] ENVIRONMENTAL SCIENCE AND MANAGEMENT,2007,10:22-2

5. 